\title{
Determinants of selectivity in Xer site-specific recombination
}

\author{
Garry Blakely and David Sherratt ${ }^{1}$ \\ Microbiology Unit, Department of Biochemistry, University of Oxford, Oxford OX1 3QU, UK
}

\begin{abstract}
A remarkable property of some DNA-binding proteins that can interact with and pair distant DNA segments is that they mediate their biological function only when their binding sites are arranged in a specific configuration. Xer site-specific recombination at natural plasmid recombination sites (e.g., cer in ColE1) is preferentially intramolecular, converting dimers to monomers. In contrast, Xer recombination at the Escherichia coli chromosomal site dif can occur intermolecularly and intramolecularly. Recombination at both types of site requires the cooperative interactions of two related recombinases, XerC and XerD, with a 30-bp recombination core site. The dif core site is sufficient for recombination when XerC and XerD are present, whereas recombination at plasmid sites requires $\sim 200$ bp of adjacent accessory sequences and accessory proteins. These accessory factors ensure that recombination is intramolecular. Here we use a model system to show that selectivity for intramolecular recombination, and the consequent requirement for accessory factors, can arise by increasing the spacing between XerC- and XerD-binding sites from 6 to 8 bp. This reduces the affinity of the recombinases for the core site and changes the geometry of the recombinase/DNA complex. These changes are correlated with altered interactions of the recombinases with the core site and a reduced efficiency of XerC-mediated cleavage. We propose that the accessory sequences and proteins compensate for these changes and provide a nucleoprotein structure of fixed geometry that can only form and function effectively on circular molecules containing directly repeated sites.
\end{abstract}

[Key Words: Site specific recombination; Xer; selectivity; recombinase]

Received November 3, 1995; revised version accepted January 20, 1996.

Site-specific recombination systems function in a range of programmed DNA rearrangements in microbes that include helping to specify developmental pathways in bacteria and bacteriophages (Sato et al. 1990; Landy 1993; Carrasco et al. 1994); determining cell type and virus host range (Zieg and Simon 1980; Hiestand-Nauer and Iida 1983; Klemm 1986; Klippel et al. 1988; Tominaga et al. 1991); processing the products of genetic transposition (Arthur and Sherratt 1979); and controling circular replicon copy number and inheritance (Summers and Sherratt 1984; Futcher 1986; Blakely et al. 1991).

Odd numbers of homologous recombination events between circular replicons during or after replication, produces dimers that need to be converted to monomers before they can be segregated normally at cell division (Austin et al. 1981; Blakely et al. 1991; Kuempel et al. 1991). Plasmid dimers can also arise as a consequence of rolling circle replication during conjugal transfer (Warren and Clark 1980; Erickson and Meyer 1993). The Xer site-specific recombination system, initially discovered by its role in plasmid ColE1 stable inheritance, also functions in the normal inheritance of the Escherichia coli chromosome and the stable inheritance of other multi-

\footnotetext{
${ }^{1}$ Corresponding author.
}

copy plasmids. Xer site-specific recombination uses two related recombinases XerC and XerD; these share $37 \%$ identity and belong to the lambda integrase family of site-specific recombinases (Blakely et al. 1993). XerC cleaves the "top" strand and XerD the "bottom" strand in in vitro recombination assays (Fig. 1A; Arciszewska and Sherratt 1995; Colloms et al. 1996; Sherratt et al. 1995; G. Blakely and D.J. Sherratt, unpubl.). Mutations in XerC, XerD, or in the chromosomal recombination site dif produce a subpopulation of cells that are filamentous and contain aberrant nucleoids. This phenotype is suppressed by mutations that abolish homologous recombination, supporting the idea that Xer recombination "undoes" the "damage" caused by homologous recombination (Blakely et al. 1991; Kuempel et al. 1991; Leslie and Sherratt 1995).

Integrase family recombinases contain four highly conserved amino acids, the RHRY tetrad (Argos et al. 1986; Abremski and Hoess 1992). The tyrosine nucleophile attacks the phosphodiester bond to be cleaved, generating a $3^{\prime}$ phosphotyrosyl protein and a free 5' hydroxyl end (Pargellis et al. 1988). The other conserved residues have been implicated in activation of the phosphodiester bonds before nucleophilic attack and religation of cleaved strands (Chen et al. 1992; Pan and Sadowski 1992). Site-specific recombination mechanisms are reviewed by Stark et al. (1992). 
A

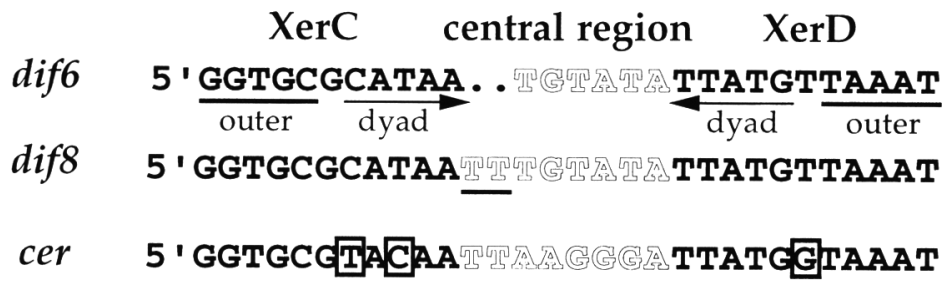

B

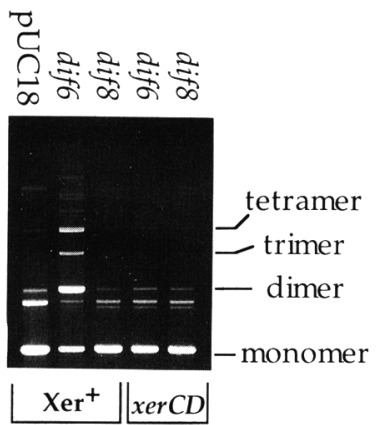

substrate

configuration

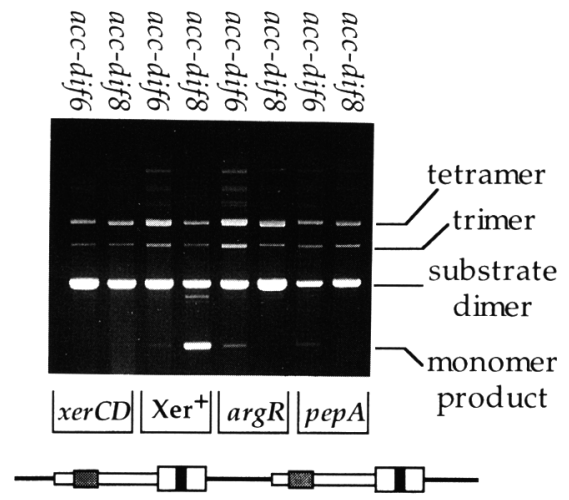

Figure 1. (A) Sequence comparison of Xer core recombination sites: dif6, from the $E$. coli chromosome; dif 8 , containing an additional TT dinucleotide (underlined) in the central region; cer, from ColE1. The base differences in left and right half-sites between dif 6 and cer are boxed. The putative outer recognition sequences are underlined, and arrows indicate the region of dyad symmetry in each half-site. $(B)$ Analysis of in vivo multimerization for plasmids containing dif6 or dif8 after transformation of monomers into $\mathrm{Xer}^{+}$or $\mathrm{xerCD}$ strains (left); dif6 was a substrate for Xer-mediated intermolecular recombination as indicated by presence of multimers; dif8 containing plasmids remained as monomers in the $\mathrm{Xer}^{+}$strain. Intramolecular resolution of plasmid dimers containing acc-dif 8 to monomer product $(\sim 30 \%$ of total DNA) only occurred in the $\mathrm{Xer}^{+}$strain (right), demonstrating its dependence on the accessory proteins ArgR and PepA. Resolution and multimerization of plasmid dimers containing acc-dif 6 was independent of accessory proteins. Note that the supercoiled tetramer band may contain some nicked dimer. Trimer formation in $\mathrm{Xer}^{-}$strains probably occur by homologous recombination within tetramers.
A specific 33-bp DNA fragment contains a functional dif site as assayed by normal chromosomal segregation and by its ability to promote Xer recombination when inserted into a plasmid (Blakely et al. 1991). dif consists of an 11-bp XerC-binding site separated by a 6-bp central region from a partially dyad symmetrical 11-bp XerDbinding site (Fig. 1A; subsequently we refer to this wildtype dif core site as dif6). The interaction of the recombinases at dif6 is highly cooperative and can lead to both intermolecular and intramolecular recombination when the site is present in a plasmid.

In contrast, recombination at natural plasmid sites (e.g., cer in ColE1 and psi in pSC101) is preferentially intramolecular, converting dimers (and higher multimers) to monomers. This selectivity for intramolecular resolution is correlated with a requirement for "accessory" proteins in addition to the recombinases and $\leqslant 200$ bp of accessory sequences adjacent to the 30-bp recombination core site (Summers and Sherratt 1984; Summers 1989; Sherratt et al. 1995). Absence of cer accessory sequences or the accessory proteins $\operatorname{ArgR}$ and PepA makes the cer core site inactive in recombination (Stirling et al. 1988, 1989). What determines whether recombination will be preferentially intramolecular and require accessory factors, or both intermolecular and intramolecular, requiring only recombinases and a recombination core site? Summers (1989) demonstrated that two variant cer sites, obtained by recombination between cer and its plasmid CloDF13 homolog parB had very different properties. Both contained the cer XerC- and XerD-binding sites, but one (the "type I hybrid"), like cer, contained an 8-bp central region (although different in sequence to cer), whereas the other (the "type II hybrid"; here designated as cer6) was deleted for a central region TT dinucleotide adjacent to the XerC-binding site and had a 6-bp central region. The type I hybrid had the recombination properties of cer, requiring accessory sequences and proteins and recombining intramolecularly. In contrast, cer6 could recombine both intermolecularly and intramolecularly when accessory proteins and sequences were absent, although it exhibited preferential intramolecular recombination when accessory sequences were present. This indicated that central region size differences could determine recombination requirements and outcomes. Nevertheless, other differences in core site sequence can influence the requirements and outcomes of recombination. For example, CloDF13 par $B$ and pSC101 psi each have a 6-bp central region, yet recombine preferentially intramolecularly when accessory sequences and proteins are present (Cornet et al. 1994; Roberts 1994; Colloms et al. 1996).

To understand more about how XerC and XerD interactions at a recombination core site determines whether accessory factors will be required and whether recombination will be preferentially intramolecular, we have used a model system that compares recombinase interactions with dif6 and a derivative of dif containing an 8 -bp central region (dif8). These sites were chosen because they have a higher affinity for XerC than cer derivatives, thus facilitating analysis of recombinase bind- 
ing. The differences in recombinase interactions with these sites provides a framework for understanding the requirements for accessory sequences and proteins in some sites and how this leads to selectivity for a particular recombination outcome.

\section{Results}

In vivo recombination properties of dif8, $a$ dif derivative containing an 8-bp central region.

Wild-type dif6, when present in a plasmid, is a substrate for Xer-mediated intermolecular and intramolecular recombination, as assayed by the conversion of plasmid monomers to multimers in a $\mathrm{Xer}^{+}$strain that is deficient in plasmid homologous recombination (Blakely et al. 1991). A comparison of the ability of dif6 and dif8 (containing an additional TT dinucleotide inserted into the central region; Fig. 1A) to act as substrates for Xer-mediated intermolecular recombination is shown in Figure 1B. Although the dif6-containing plasmid was an efficient substrate for intermolecular recombination $\mid \sim 70 \%$ of the DNA being converted from monomers to multimers after $\sim 40$-cell generations). The dif8-containing plasmid produced the same levels of monomers ( $\sim 90 \%)$ in $\mathrm{Xer}^{+}$and $\mathrm{Xer}^{-}$strains. Therefore, dif8 cannot function as an Xer recombination core site.

To determine whether dif 6 and dif 8 can respond to accessory sequences and proteins, derivatives were constructed that contained the cer accessory sequences upstream of the XerC-binding sites. We refer to these sites as acc-dif6 and acc-dif8. To test these sites for intramolecular resolution, dimers of plasmids containing either acc-dif6 or acc-dif 8 were used to transform $\mathrm{Xer}^{+}$, $x e r C D, \arg R$, and pepA strains and the resulting plasmid DNAs were analyzed. Whereas the $a c c$-dif6 dimers were substrates for both intermolecular and intramolecular Xer recombination that was independent of PepA and ArgR (Fig. 1B), acc-dif8 only recombined intramolecularly in a reaction that requires PepA, ArgR, XerC, and XerD (Fig. 1B). This is similar to the behavior of cer, although note that resolution of acc-dif8 dimers was incomplete $(\sim 30 \%$ of the DNA was monomeric after $\sim 40$ generations), compared to the $\geqslant 80 \%$ resolution of cer dimers in similar experiments (Summers and Sherratt 1984). This incomplete resolution could be a consequence of a 1-bp insertion between the core site and accessory sequences in acc-dif8 compared to cer /see Materials and methods).

Taken together these results show that the addition of 2 bp between the recombinase binding sites of dif 6 destroys the site's ability to act as a core site for Xer-mediated recombination, both for intermolecular and intramolecular events. Recombination proficiency is only restored to dif 8 when accessory sequences and proteins are present; then resolution selectivity is imposed on the site. The addition of accessory sequences to dif 6 appeared to have little effect on its ability to recombine and did not impose directionality to its reactions. This contrasts to the properties of cer6, which shows resolution selectivity in the presence of ArgR and PepA, but recombines intermolecularly and intramolecularly in their absence (Summers 1989). Therefore, the sequence differences in the core sites of cer and dif may also play a role in determining whether a core site responds to accessory sequences and proteins.

\section{Recombinase binding to dif 6 and dif 8}

Because binding of XerC and XerD to dif is known to be highly cooperative (Blakely et al. 1993), we believe it likely that the proteins interact when bound to their target. The addition of an intervening $2 \mathrm{bp}$ to B-form DNA will rotate the relative positions of the two recombinase binding sites by $\sim 69^{\circ}$ and separate them by $6.8 \AA$. Such rotation and spatial separation may be expected to alter the ability of recombinases to interact across the intervening DNA (Mao et al. 1994).

Differences in cooperative binding of the recombinases to dif 6 and dif 8 were analyzed using gel retardation to measure the binding of XerC and XerD to dif-containing fragments as a function of recombinase concentration. The apparent equilibrium dissociation constant $\left(K_{\mathrm{d}}\right)$ for each of the recombinases binding individually to dif6 and dif 8 were similar; $\sim 177 \mathrm{~nm}$ for XerC binding and $\sim 6 \mathrm{nM}$ for XerD binding, indicating that the 2-bp insertion does not influence the binding of either recombinase alone and confirming our earlier observation that XerD binds with a higher affinity than XerC. Titration of XerC in the presence of constant XerD (250 nM) for the two sites indicated that the apparent $K_{\mathrm{d}}$ of XerC for a dif6/XerD complex was $\sim 0.5 \mathrm{nM}$ whereas the apparent $K_{\mathrm{d}}$ of XerC for a dif8/XerD complex was $\sim 2.4 \mathrm{nM}$ (Fig. 2A). This indicates a reduction in cooperativity index from $\sim 300$ fold for dif6 to 60 - to 75 -fold for dif8, a reduction in overall affinity of $\sim 5$-fold.

Complexes of XerC and XerD with dif8 migrate more slowly than complexes with dif6 (see Fig. 2B), indicating that the dif8/XerC/XerD complex has undergone additional bending (Wu and Crothers 1984). This is similar to the increased mobility of a recombinase/cer complex relative to a recombinase/dif complex (Blakely et al. 1993). Because of this difference, we used circular permutation experiments to analyze the nature of potential alterations in bending induced by XerC and XerD. A bend in the center of a fragment will reduce its mobility in relation to the same bend at the end of the fragment (Wu and Crothers 1984; Lane et al. 1992).

$\mathrm{XerC}$ bound alone to either dif6 or dif8 induced a similar small bend $\left[\sim 25^{\circ}\right.$; calculated using the method of Thompson and Landy (1988)]. XerD induced a larger bend in both sites $\left(\sim 40^{\circ}\right)$. Measurement of relative mobilities of dif6 and dif 8 complexes with XerC and XerD shows that the overall bend angle (note this is a composite angle because of bends induced by XerC and XerD) was greater for dif $8\left(60^{\circ}-65^{\circ}\right)$ than for dif6 $\left(40^{\circ}-45^{\circ}\right)$. The fact that there may be more than one bend present precludes us from estimating a meaningful bend center.

We offer three explanations for the differences in overall bend angles for dif6 and dif8: (1) the individual XerC 
A

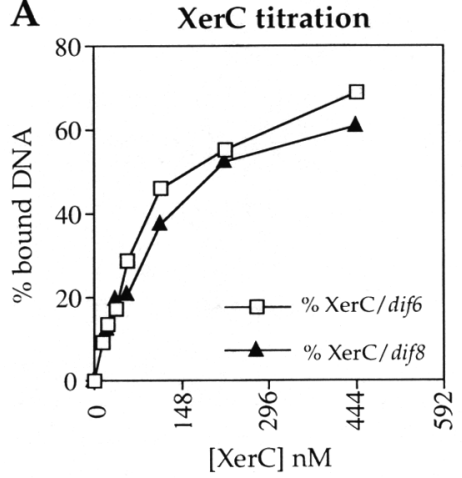

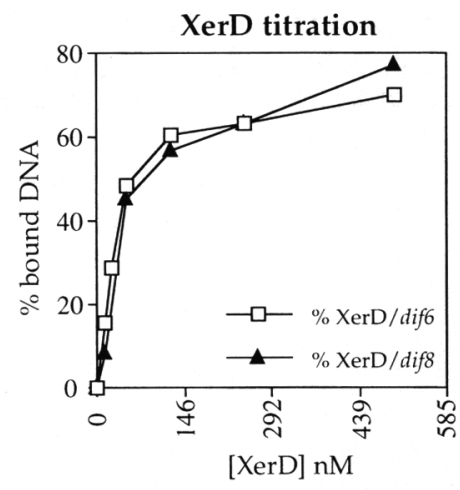

Titration of XerC against XerD/DNA

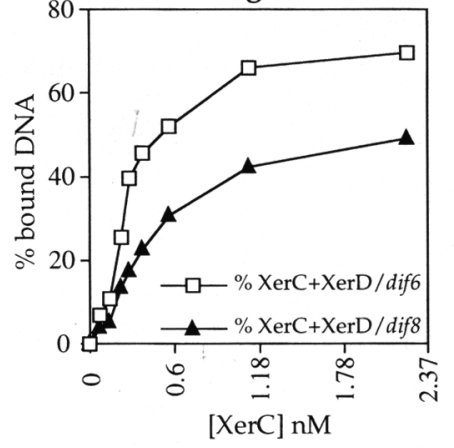

B
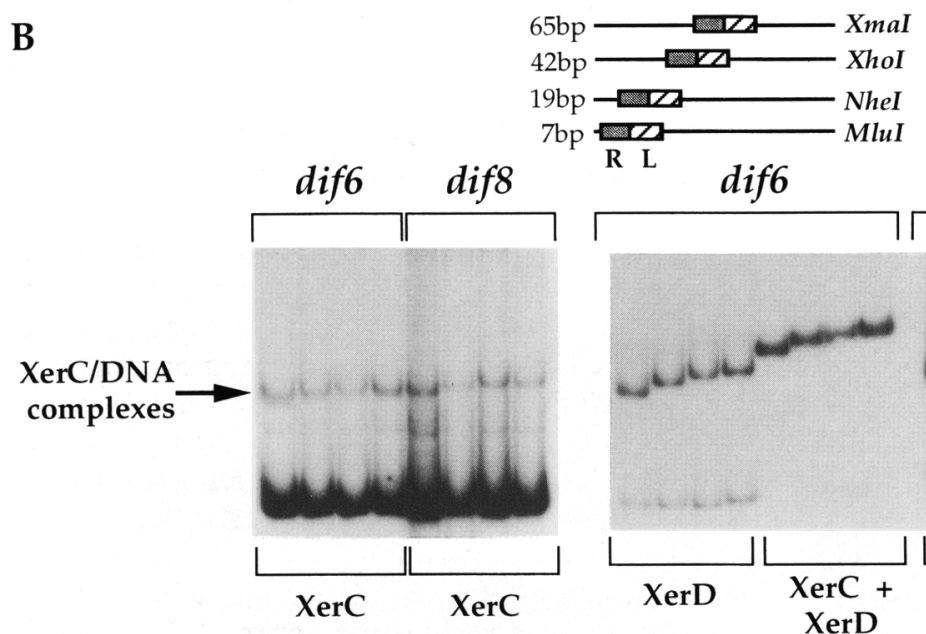

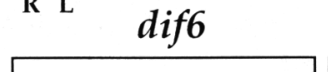

dif8

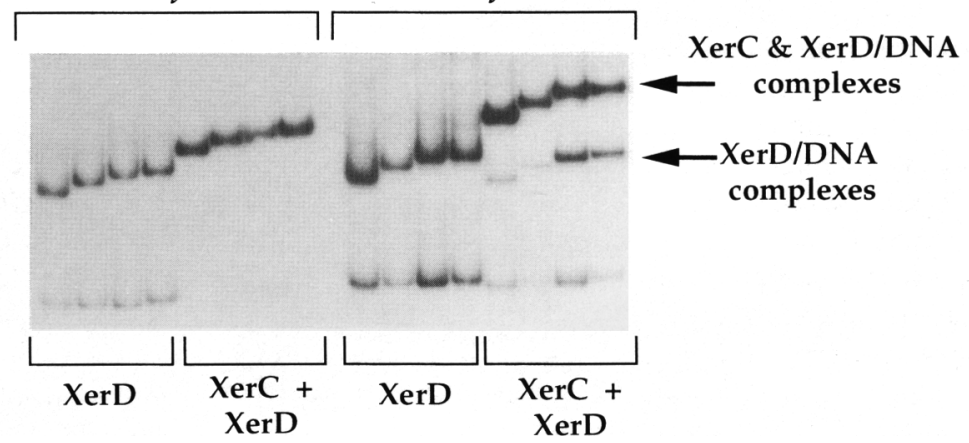

Figure 2. (A) Comparison of binding curves for titration of each recombinase with core recombination sites demonstrates similar apparent affinities for XerC and XerD binding to left and right half-sites, respectively, for both diff and dif8. In the presence of XerD and higher concentrations of $\operatorname{XerC}(\geqslant 100 \mathrm{nM}),>90 \%$ of DNA is bound. $(B)$ Bending analysis using gel retardation for recombinases bound to DNA fragments containing either dif6 or dif 8 generated by restriction enzyme digestion of plasmids based on pBEND2. Restriction fragments generated by MluI, NheI, XhoI, and XmaI, from left to right for each set of four reactions. Distances from the right half-site to the end of the DNA fragments are indicated. Note that the XerC/XerD/dif 8 complex is more retarded than the equivalent dif6 complex, indicating an altered geometry.

and $\mathrm{XerD}$ bends in difo and dif8 remain the same, with the $69^{\circ}$ relative rotation of binding sites in dif8 producing a phasing change between bends induced individually by each recombinase; (2) an additional bend is introduced in the dif8 DNA either as a direct consequence of the TT dinucleotide insertion, or because of changes in recombinase interactions induced by the spacing change; and (3) the individual bends induced by XerC and XerD are different in the two sites as a consequence of changes in DNA sequence or recombinase interactions. These explanations are not mutually exclusive and we cannot distinguish between them on the basis of these experiments. Altered geometry, leading to reduced electrophoretic mobility, appears to be diagnostic for recombination sites that show resolution selectivity.

\section{In vitro XerC-mediated cleavage assay}

The inability of dif 8 to undergo recombination, without the presence of accessory sequences, suggested that this site was defective in one or more of the following steps: synapsis, strand cleavage, and strand exchange. Strand cleavage can be assayed by the accumulation of recombinase-DNA covalent complexes in linear duplex "suicide" substrates that contain a nick three nucleotides 3 ' from the recombinase-mediated cleavage site. Cleavage releases a trinucleotide, thus trapping the covalent protein-DNA complex because there is no adjacent $5^{\prime} \mathrm{OH}$ that can attack the phosphotyrosine and reverse the reaction (Nunes-Duby et al. 1987; Sherratt et al. 1995). Suicide substrates based on dif6 and dif 8 were used to compare the cleavage efficiences between the two sites and determine whether dif8 is a substrate for XerC cleavage. A cer core site substrate was also used to ascertain the ability of XerC to cleave at another site that contained an 8-bp central region.

Defined concentrations of recombinases that ensured saturation of binding sites /confirmed by gel retardation; $1 \mu \mathrm{M}$ each of XerC and XerD| were added to top strand suicide substrates containing a nick three nucleotides $3^{\prime}$ 
from the XerC cleavage site and the amount of covalent XerC-DNA complex determined by electrophoresis through polyacrylamide containing $0.1 \%$ SDS. Sixty to $70 \%$ of dif6 DNA was converted to XerC covalent complexes in a 60-min reaction (Fig. 3). A weak second band running above the XerC/dif6 covalent complex is possibly a strand transfer product resulting from a nucleophilic attack of a 5' $\mathrm{OH}$ end of an oligonucleotide from a second covalent complex. Reactions containing an XerC-maltose-binding protein fusion confirm that top strand cleavages were mediated by $\mathrm{XerC}_{\text {; }}$ note the greater retardation attributable to the size of the fusion protein. Half molecule products resulting from XerDmediated bottom strand cleavage of dif $6 / \sim 1 \%$ of total DNA) were also detectable as faint bands running below the substrate. The amount of covalent complex produced by XerC cleavage of dif 8 in a 60 -min reaction was $2 \%-$ $3 \%$ of total DNA, which was comparable to the amount of complex formed with the cer core sequence. Therefore, inefficient XerC cleavage of a core recombination site correlates with resolution selectivity and requirement for accessory factors. Note that the cleavage substrates used here may not assay every cleavage event; the trinucleotide generated by recombinase cleavage could remain bound to the duplex as a consequence of recombinase/DNA interactions and therefore, be available for reversal of the reaction. Nevertheless, we believe that comparative measurements of cleavage at dif6 and dif 8 do indicate a real difference in cleavage efficiency.

Comparison of XerC covalent complexes formed with a cer6 substrate and a derivative containing an additional TT dinucleotide at the $3^{\prime}$ end of the central region confirmed that it is the 8-bp central region that causes the reduction in detectable cleavages and not the addition of the TT bases adjacent to the XerC cleavage position /data not shown). We conclude that the ability of XerC to cleave DNA is strongly influenced by the interactions between XerC and XerD and the geometry of the recombinase-DNA complex.

\section{Altered protection of recombinase/dif8 complexes from attack by the chemical nuclease $\mathrm{OP}-\mathrm{Cu}$}

The 1, 10-phenanthroline-copper (OP-Cu) reagent is a chemical nuclease that interacts with DNA through the minor groove, where it initiates an oxidative attack on the Cl-hydrogen of deoxyribose leading to cleavage of the DNA backbone (Sigman et al. 1991; Spassky et al. 1988). Resistance to cleavage is caused by blocking access to the DNA either as a result of protein steric hindrance or altered geometry that narrows the minor groove. Enhanced cleavage by OP-Cu results from altered DNA geometry that leads to a widened minor groove.

An OP-Cu footprint for the recombinases bound individually to dif6 has shown that XerC binds to the left half-site and XerD binds to the right half-site (Blakely et al. 1993; see Fig. 1A). Binding of both recombinases to dif6 results in almost complete occlusion of the OP-Cu reagent from the core site, giving protection of $28 \mathrm{bp}$ (Fig. 4). Analogous footprinting reactions of recombinases bound at dif 8 demonstrated that although protection of the right half-site resembled dif6, almost all of the central region and the innermost AA dinucleotide of the left half-site had become susceptible to nuclease attack (Fig. 4). Cleavages were enhanced at deoxyribose groups corresponding to base positions 2 and 3 on the top strand and -1 and 1 on the bottom strand (Fig. 4); these posi-
Figure 3. XerC-mediated cleavage at core recombination sites tested in vitro using radiolabeled (*) suicide substrates containing a nick in the top strand of the central region. Reactions $\left(37^{\circ} \mathrm{C} ; 60 \mathrm{~min}\right)$ containing XerC + XerD $(C D)$ or XerC-maltose-binding protein +XerD $\left(C^{M} D\right)$ were electrophoresed through $6 \%$ polyacrylamide containing $0.1 \%$ SDS and amount of substrate converted to covalent recombinase/DNA complex was quantified. The top strand of dif6 was cleaved efficiently by $\mathrm{XerC}$, whereas cleavage of top strands for both dif8 and cer was substantially lower. Increased retardation of the recombinase/ DNA complex in reactions containing XerC-MBP demonstrates that XerC was responsible for top strand cleavage. The half molecules visible below the substrate are generated by XerD cleavage of the bottom strand producing a double strand break.

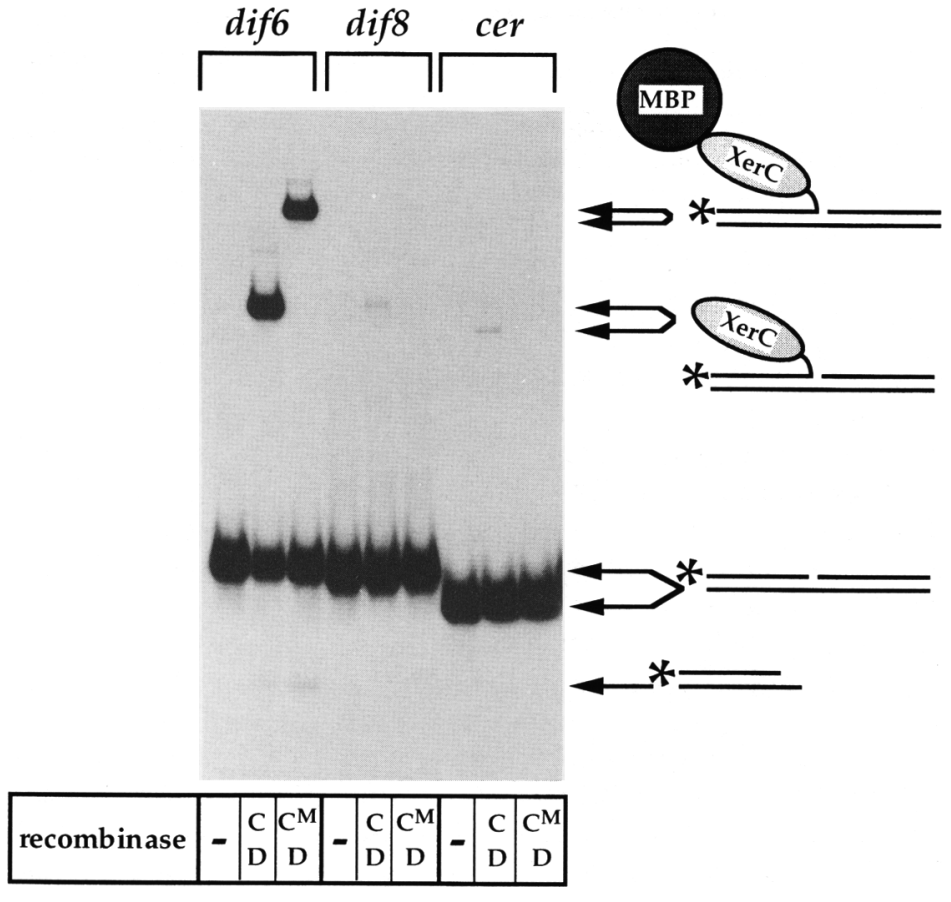



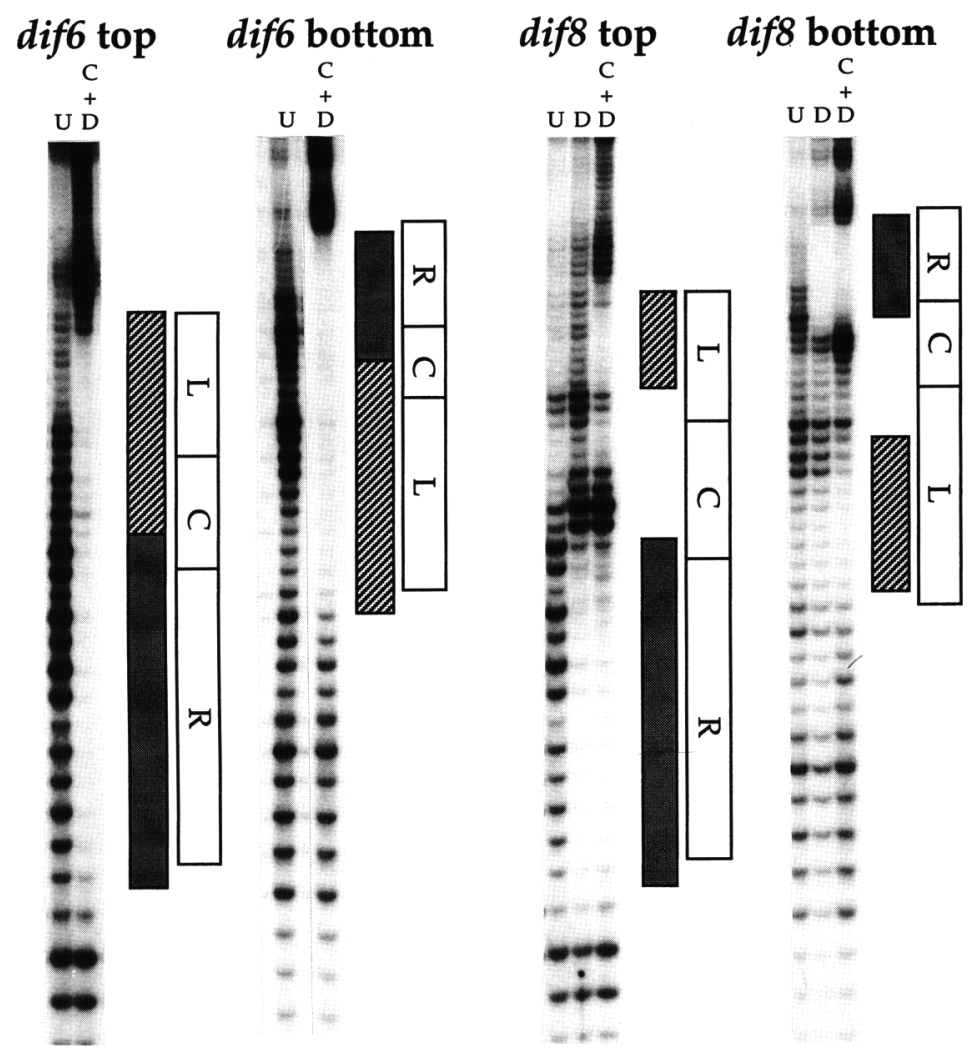

Figure 4. In situ 1,10-phenanthroline-copper footprinting was used to compare interactions of recombinases with dif6 or dif8. Reactions containing $\mathrm{XerC}+\mathrm{XerD}(\mathrm{C}+\mathrm{D})$, XerD alone (D), or unbound DNA $(\mathrm{U})$ are shown with regions protected from $\mathrm{OP}-\mathrm{Cu}$ cleavage indicated as shaded boxes. Diagrammatic representations of core recombination sites are included adjacent to the autoradiographs and show left (L) and right (R) half-sites and central region (C). Sequences protected by XerC and XerD are shown at the bottom. Left and right half-sites are shown in boldface type, and the central region sequence is indicated as hollow lettering. Note the loss of protection for top and bottom strands of the dyad sequence in the left half-site and sensitivity of the central region in dif8. Arrows on the dif 8 sequence represent enhanced OP-Cu cleavages. Sequence coordinates are according to Blakely et al. \{1993\}.

tions represent the symmetrical cleavages generated by the OP-Cu complex, where the nearest residue on the opposite strand is $\mathrm{n}+2$ across the minor groove (Conner et al. 1984). Binding of XerD alone to dif6 (Blakely et al. 1993) and dif8 (Fig. 4) also leads to some enhanced cleavage in the top strand central region adjacent to the right half-site. In contrast, there are no enhancements on the bottom strand in the presence of XerD alone. The altered patterns of protection and enhancements for the XerC/ $\mathrm{XerD} /$ dif 8 complex are indicative of additional DNA distortions, which may contribute to the overall geometry of the complex as measured by electrophoresis. We also note that naked dif6 and dif8 DNA were not uniformily sensitive to OP-Cu cleavage; the central region and the dyad sequences were cleaved more readily than adjacent sequences (Fig. 4); we do not know if this is relevant to core site function.

The loss of protection for the innermost AA of the XerC-binding site, adjacent to where XerC cleaves (Fig. 4 ), suggests a reduced interaction of XerC with this region of the dif8 DNA or a slight widening of the minor groove. This region of the XerC-binding site shows dyad symmetry with the inner region of the XerD-binding site and is presumably the recognition sequence for the catalytic domain of XerC. An altered disposition of XerC catalytic residues in relation to the strand cleavage position may explain the low levels of XerC-mediated cleavage observed with dif 8 suicide substrates. 


\section{Hydroxyl radical footprinting of dif6 and dif8}

The small diffusible hydroxyl radical, which cleaves DNA with little or no sequence specificity, can be used to detect DNA deformation and protein binding (Tullius and Dombroski 1985, 1986). Gel retardation was used to separate recombinase-bound from unbound DNA after treatment with the reagents; footprints were only detected for XerD and not XerC when reactions were performed without gel retardation (data not shown); presumably because XerC binding is weak compared to XerD binding (this study; Blakely et al. 1993; Blakely and Sherratt 1994).

Large regions of protection from hydroxyl radical cleavage were detected on top and bottom strands for both dif 6 and dif 8 ; there were no regions of strongly enhanced cleavage. Most of the central region of dif 6 was protected from cleavage (Fig. 5), again suggesting that the two recombinases come into close contact across the central region of this site. In contrast, the middle of the dif 8 central region is sensitive to hydroxyl radicals, further supporting the view of reduced recombinase contact in this region of dif8. The regions of dyad symmetry flanking the central region were protected on both strands, although the dif $8 \mathrm{XerC}$-binding site inner region was less protected than that of dif6, reinforcing the other evidence for reduced contact of XerC with this region of dif8 (Fig. 5).

Protection of the three outer nucleotides of the XerC (top strand) and XerD (bottom strand) binding sites de-
Figure 5. Hydroxyl radical footprints of $\mathrm{XerC}+\mathrm{XerD}$ bound to dif6 and dif8. Regions of core sites protected from cleavage in the presence of $\mathrm{XerC}+\mathrm{XerD}\{\mathrm{C}+\mathrm{D}\}$, compared to unbound DNA (U), are indicated adjacent to the autoradiographs as are the relative positions of the core sites. Protection of outer sequences on both dif6 and dif 8 denote contacts that may be involved in recognition specificity. Note reduced protection of the dyad portion of the left half-site sequence on both top and bottom strands, which is again indicative of reduced XerC interactions. The insets at the bottom of the figure illustrate that $\mathrm{H}_{2} \mathrm{O}_{2}$ can act as a nucleophile to mediate cleavage when a specific phosphodiester is activated by XerC and XerD. These cleaved products only occur when dif6 is bound by $\mathrm{XerC}+\mathrm{XerD}$. Maxam-Gilbert $\mathrm{A}+\mathrm{G}$ sequencing reactions $(A+G)$ are included for each set of footprints.

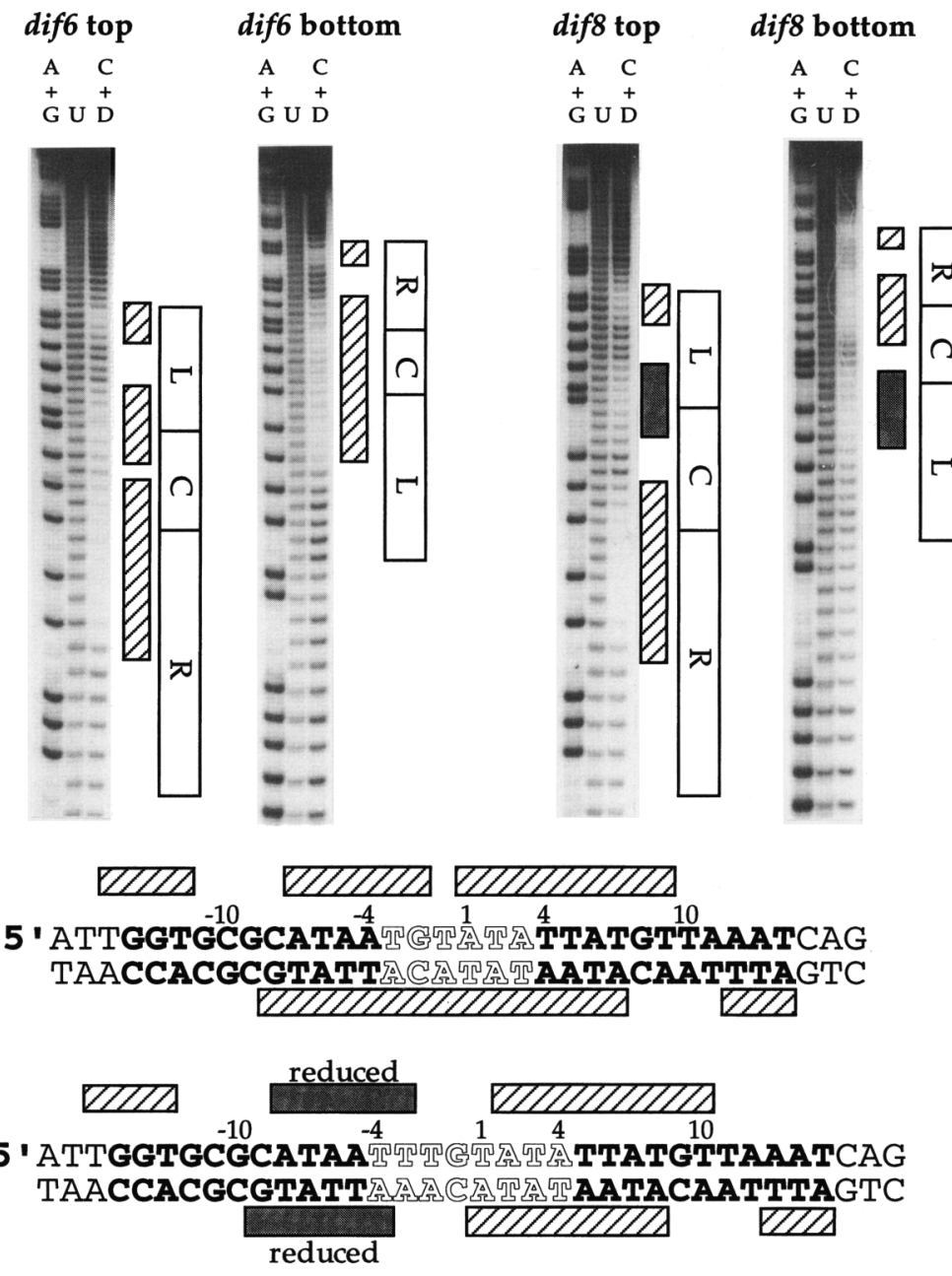

Top strand cleavage position

Bottom strand cleavage position
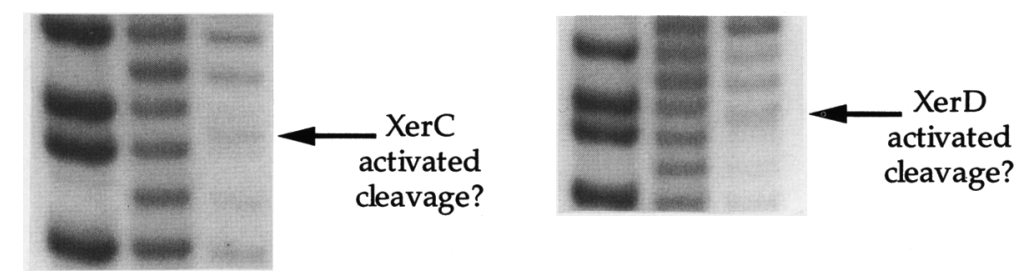
lineate the outermost backbone contacts of the recombinases. These nucleotides along with the two immediately inside them have been implicated in contacts with XerC and XerD and in providing binding specificity (Blakely and Sherratt 1994). The 5' TTT sequence in the bottom strand of the right half-site may be contacted by XerD through the major groove, as suggested by $\mathrm{KMnO}_{4}$ interference footprinting (Blakely and Sherratt 1994). The hydroxyl radical protection pattern for the right half-site also shows similarity to the ethylation interference footprint for XerD binding (Blakely and Sherratt 1994), demonstrating the extensive interactions of XerD with the DNA backbone.

An additional weak band was observed in both top and bottom strand footprints (Fig. 5, insets). We believe that these correspond to cleavage mediated by $\mathrm{H}_{2} \mathrm{O}_{2}$ as the nucleophile and activated by XerC (top strand) and XerD (bottom strand); the $3^{\prime}$ end-labeled products should have $5^{\prime} \mathrm{OH}$ groups, explaining why migration is retarded by approximately half a base pair on these sequencing gels (Blakely and Sherratt 1994). Kimball et al. (1993) reported that hydrogen peroxide can act as an exogenous nucleophile for cleavage of FRT DNA in the presence of FLP recombinase.

\section{Discussion}

Communication between distant DNA segments is important in a variety of biological reactions that include control of transcription, homologous recombination and gene conversion, mismatch repair, and site-specific recombination. In many of these interactions it is important for normal biological function that "correct interactions" occur to the exclusion of "incorrect interactions." For example, in activation of transcription, a gene's enhancers and bound transcription factors must activate expression of that gene but not that of neighboring genes (discussed in Corces 1995). Similarly, site-specific recombination can be limited to a pair of identical recombination sites in a particular configuration (discussed in Stark and Boocock 1995). A novel and intriguing feature of the Xer site-specific recombination system is that it can function with or without selectivity for a particular configuration of recombination sites, depending on the structure of the recombination site. This dual behavior is a valuable resource for analyzing the selectivity mechanism, as well as for providing insight into how selectivity can arise.

On all natural plasmid substrates for Xer recombination tested, recombination is preferentially intramolecular, converting multimers (that can arise by homologous recombination or by rolling circle conjugal transfer) to monomers. We believe that this resolution selectivity results from the use of a "topological filter" that ensures that productive synaptic complexes, which must have a fixed protein/DNA geometry, can only form and recombine on supercoiled substrates containing two directly repeated recombination sites (discussed in Boocock et al. 1987; Stark et al. 1989; Stark and Boocock 1995; Col- loms et al. 1996). It is difficult to see how such a topological filter could operate to differentiate dif6 sites on two separate monomeric chromosomes from two dif sites separated by $4.7 \mathrm{Mb}$ on a dimeric chromosome. It is not surprising, therefore, to find that recombination at dif6 does not show resolution selectivity, at least in the plasmid assays that we have available.

In the model system used here, a 2-bp increase in central region spacing from dif6 to dif8, converts a core site that recombines intermolecularly and intramolecularly in vivo to one that shows preferential intramolecular resolution and requires adjacent accessory sequences and proteins. This mirrors precisely the in vivo recombination differences between dif6 and the natural plasmid sites. The different in vivo recombination properties of dif 6 and dif 8 are correlated with changes in recombinase interactions with their targets, the geometry of the recombinase/core site complex, and the efficiency of XerC-mediated cleavage of DNA.

The simplest hypothesis that we can offer to explain the in vivo recombination properties of dif 6 and dif 8 is that a dif6/recombinase complex has the geometry and stability to be able to form a recombinational synapse with an identical partner complex using recombinaserecombinase interactions. Such synaptic structures may form irrespective of the configuration of the two sites, thus permitting intramolecular and intermolecular recombination. In dif8, as a consequence of changed core site/recombinase geometry and altered recombinase binding, catalytically active synapsed recombinase/core site complexes may be unable to form on any configuration of two sites. The addition of accessory sequences and the provision of accessory proteins that interact with these sequences could provide extra nucleoprotein "glue" that allows the formation of a catalytically proficient synaptic complex. We would expect this complex to have a fixed local geometry; this dictates that it can only form and function on directly repeated sites in the same molecule (e.g., see Stark and Boocock 1995).

The reduced XerC cleavage of dif8 (and other sites that show resolution selectivity) on the linear suicide substrates used here, and on synthetic Holliday junctioncontaining substrates (L.K. Arciszewska and D.J. Sherratt, unpubl.) suggests that the accessory sequences and proteins are not only required for synapsis of two dif 8 sites, but may be necessary to alter the geometry of the core site/recombinase complex within the whole synaptic structure. We propose that this allows XerC to interact correctly with its binding site, so that strand exchange can be initiated. From in vitro recombination experiments on supercoiled substrates containing two directly repeated plasmid recombination sites, we know that XerC strand exchange precedes XerD exchange and that the synaptic complexes containing these sites must have a fixed local geometry (Colloms et al. 1996; S. Colloms, J. Bath, and D.J. Sherratt, unpubl.). However, as yet, we are not able to assay synapse formation and structure biochemically and therefore, cannot test directly the above hypothesis. Note that we do not believe recombination on linear suicide substrates requires syn- 
apsis of two core site fragments (G. Blakely and D.J. Sherratt, unpubl.).

It seems likely that the inefficient cleavage of dif 8 by $\mathrm{XerC}$ is a consequence of the altered XerC interactions with the inner dyad (CATAA) portion of the left half-site when $\mathrm{XerD}$ is present. The alterations, which probably result from the changed geometry of the recombinase/ core site complex, are indicated by reduced protection of the CATAA dyad sequence by XerC with both footprinting reagents and possibly an additional bend in dif 8 as suggested by the enhanced OP-Cu cleavages at the $3^{\prime}$ end of the central region adjacent to the right half-site. However, the footprinting data do not provide additional insight into the structural basis for and positions of all the bends in dif 6 and dif8.

In dif6, XerC and XerD together protect all of the central region from chemical attack by OP-Cu and also largely protect it from hydroxyl radical attack. The footprints shown here and earlier (Blakely et al. 1993) demonstrate that each recombinase spans the central region strand that they cleave (Fig. 4), which suggests that XerC and XerD may be in close contact with each other in this region. Such contacts may in part be responsible for the cooperative interactions between the two recombinases. In contrast, the dif 8 central region is sensitive to cleavage by both reagents when XerC and XerD are bound, demonstrating that specific domains of the recombinases have moved away from the central region DNA possibly to facilitate formation of cooperative protein/ protein interactions. Although we have no firm evidence that indicates which part of each recombinase is in contact with the central region, we believe that one or both conserved arginines implicated in phosphodiester activation (Argos et al. 1986; Abremski and Hoess 1992; Chen et al. 1992) will interact with the CATAA dyad motif before XerC and XerD cleavage. Therefore, regions close to, or including, domain II may contact the central region. For XerD we have demonstrated directly an interaction between Arg247 and the phosphodiester that is cleaved (G. Blakely and D. Sherratt, unpubl.). This conserved domain II arginine, which is involved in phosphodiester activation, is present in regions of XerC and XerD that are predicted by computer analysis to have alpha helical secondary structure (G. Blakely and D. Sherratt, unpubl.). Such interaction of a helical XerC domain II with the major groove of the left half-site dyad could allow cleavage by $\mathrm{OP}-\mathrm{Cu}$ and protection against hydroxyl radical cleavage. Similar findings were obtained with $E c o$ RI; major groove interactions of a helical domain containing the catalytic site with the GAA recognition half-site gave protection against hydroxyl radical cleavage but allowed complete accessibility to OP$\mathrm{Cu}$ (Kuwabara et al. 1986; McClarin et al. 1986).

The four- to fivefold difference in relative affinities of recombinase binding to dif 6 and dif 8 is not a dramatic change. Comparison of affinities for dimers of phage HK022 cI repressor bound at sites separated by variable spacing demonstrated that a 2-bp insertion led to a 1000 fold decrease in cooperativity (Mao et al. 1994). Lack of flexibility in the protein structure was used to explain this cooperativity loss. The relatively small reduction in cooperative interactions between XerC and XerD bound at dif 8 could suggest that one or both of the proteins contain a flexible region near the presumptive heterodimerization domain. Alternatively, distortion in the DNA induced by recombinase binding, rather than protein flexibility, could explain the relatively small loss in cooperativity. The differences in recombinase-induced bending are consistent with this idea, as is the gain in chemical sensitivity in the dif 8 central region and the left half-site. If XerC is "anchored" to the outer specificity region of its binding site (Fig. 1A) and the recombinase is fairly inflexible, then it may be pulled away from the dyad and central region of dif8 DNA causing altered bending to maintain XerC/XerD contacts.

Our demonstration that differences in spacing between binding sites for two interacting proteins can give rise to biologically different outcomes is not unique. For example, the steroid receptor-binding sites can have variable spacing ranging from 1 to $5 \mathrm{bp}$. Identical binding sites with a 1-bp spacing give a retinoid $X$ receptor-binding site, whereas a 4-bp spacing produces a $\mathrm{T} 3$ response element (Shulemovich et al. 1995). Similarly, it seems possible that topological filtering of the type that we believe is involved in resolution selectivity described here (discussed in Stark and Boocock 1995) is widely used to discriminate between arrays of distant sites containing bound proteins in complexes of fixed architecture and geometry. This may ensure that enhancers and their bound transcription factors activate appropriate genes at the correct time during differentiation and development (for review, see Corces 1995; Chi et al. 1995; Wijgerde et al. 1995).

\section{Materials and methods}

Strains and plasmids

All strains were derivatives of E. coli K12 AB1157 (Bachman 1972). The $\mathrm{Xer}^{+}$strain DS941 is $\mathrm{AB} 1157$ recF $\mathrm{lacI}^{q}$ lacZDDM15; DS9009 is DS941 xerD2::Tn10-9 xerCY17; DS956 is DS941 argR::fol; DS957 is DS941 pepA::Tn5 (Stirling et al. 1988; Colloms et al. 1990; Blakely et al. 1993; McCulloch et al. 1994). Construction of plasmid pMIN33 containing dif6 has been described (Blakely et al. 1991). Synthetic oligonucleotides corresponding to the dif 8 sequence were annealed and ligated into the $\mathrm{XbaI}$ and SalI restriction sites of pUC18 to give plasmid pGB300. Derivatives of core recombination sites with accessory sequences were constructed by digestion of the cer containing plasmid pKS492 (Stirling et al. 1988) with MluI followed by Klenow treatment and subsequent digestion with EcoRI; this 193-bp fragment was ligated to either pMIN33 or pGB300, which had been digested with $X b a \mathrm{I}$, treated with Klenow, and then digested with EcoRI to give pGB305 and pGB306, respectively. Plasmids for circular permutation analysis were generated by ligating oligonucleotides containing dif6 or dif8 into $X b a I$ and Sall sites of pBEND2 (Kim et al. 1989). DNA sequences were confirmed using the dideoxy chain termination sequencing method.

DNA methods

Routine methods for DNA isolation and in vitro manipulation 
were used (Sambrook et al. 1989). Oligonucleotides were labeled using T4 polynucleotide kinase with $\left[\gamma^{-32} \mathrm{P}\right] \mathrm{ATP}$. Restriction fragments for footprinting and $\mathrm{pBEND} 2$ analysis were labeled using the Klenow fragment of DNA polymerase with the appropriate $\left[\alpha^{-32} \mathrm{P}\right] \mathrm{dNTP}$ as described previously (Blakely and Sherratt 1994).

\section{Oligonucleotides}

Synthetic oligonucleotides (compare to difo in Blakely et al. 1991) containing the dif8 sequence were as follows: top strand, 5'-CTAGAATTGGTGCGCATAATTTGTATATTATGTTAAATCAG; and bottom strand, 5'-TCGACTGATTTAACATAATATACAAATTATGCGCACCAATT.

\section{Gel retardation and proteins}

Binding of XerC and XerD to DNA fragments was performed in buffer containing $20 \mathrm{~mm}$ Tris- $\mathrm{HCl}(\mathrm{pH}$ 7.5), $50 \mathrm{~mm} \mathrm{NaCl}, 1 \mathrm{~mm}$ EDTA, $1 \mathrm{mg} / \mathrm{ml}$ of poly[d(I-C)] and 0.1 pmoles of labeled DNA for $10 \mathrm{~min}$ at $37^{\circ} \mathrm{C}$ before electrophoresis through $6 \%$ polyacrylamide in $1 \times$ TBE buffer at $4^{\circ} \mathrm{C}$. Bend angles were determined from $8 \%$ polyacrylamide gels using the method of Thompson and Landy (1988). XerC and XerD were purified by fast protein liquid chromatography on Mono S columns as described previously (Blakely et al. 1993). Maltose-binding protein fusions were prepared as described in Arcizewska and Sherratt (1995). We have not determined what proportion of our recombinase preparations are active in binding or recombination. Apparent $K_{\mathrm{d}} \mathrm{s}$ were determined as the protein concentration that bound $50 \%$ of DNA substrate.

\section{In vitro cleavage assays}

Suicide substrates were constructed by annealing two oligonucleotides, corresponding to left and right halves of top strand core sites flanked by pUCl 8 sequences, to an oligonucleotide corresponding to the bottom strand. Resulting double-stranded DNA thus contained a nick in the top strand of the core site central region. XerC and XerD (final concentration of $1 \mu \mathrm{M}$ for each/ were added to 0.1 pmole of labeled substrate in binding buffer (as above) and incubated at $37^{\circ} \mathrm{C}$ for $1 \mathrm{hr}$. Reactions were split in two; one portion was electrophoresed through $6 \%$ polyacrylamide under nondenaturing conditions in $1 \times$ TBE buffer, the other portion being treated with SDS and EDTA to give final concentrations of $0.1 \%$ and $1 \mathrm{~mm}$, respectively. SDS treated samples were then electrophoresed through $6 \%$ polyacrylamide $+0.1 \%$ SDS in $1 \times$ TBE buffer containing $0.1 \%$ SDS.

\section{In situ OP-Cu footprinting}

End-labeling of top and bottom strands of dif 6 or dif 8 was performed using the 67-bp HindIII-KpnI and the 73-bp EcoRI-SphI fragments, respectively, from plasmids pMIN33 and pGB300. Scaled-up binding reactions containing $3.4 \mu \mathrm{g} / \mathrm{ml}$ of total protein were electrophoresed through $6 \%$ nondenaturing polyacrylamide, with retarded complexes detected by autoradiography at $4^{\circ} \mathrm{C}$ (Blakely et al. 1993). Excised gel fragments were immersed in $100 \mu \mathrm{l}$ of $50 \mathrm{~mm}$ Tris- $\mathrm{HCl} / \mathrm{pH} 8.0\}$ before addition of $10 \mu$ l of the OP-Cu mix $(2.0 \mathrm{~mm} 1,10$-phenanthroline and 0.45 $\mathrm{mM} \mathrm{CuSO}_{4}$ ) and $10 \mu \mathrm{l}$ of $58 \mathrm{~mm}$ mercaptoproprionic acid (Sigman et al. 1991); the mixture was gently vortexed and incubated at room temperature for $15 \mathrm{~min}$. The reaction was terminated by addition of $20 \mu \mathrm{l}$ of 2,9-dimethyl-1,10-phenanthroline. DNA fragments were eluted and precipitated before electrophoresis through $20 \%$ sequencing gels.

\section{Hydroxyl radical footprinting}

Binding reactions containing glycerol-free recombinases $(\sim 3$ $\mu \mathrm{g} / \mathrm{ml})$ in $70 \mu \mathrm{l}$ of $20 \mathrm{~mm}$ Tris- $\mathrm{HCl}(\mathrm{pH} 7.5), 50 \mathrm{~mm} \mathrm{NaCl}$ were incubated at $37^{\circ} \mathrm{C}$ for $10 \mathrm{~min}$. Footprinting reactions, carried out at room temperature for $2 \mathrm{~min}$, contained reagents at a final concentration of $120 \mu \mathrm{M}\left[\left(\mathrm{NH}_{4}\right)_{2} \mathrm{Fe}\left(\mathrm{SO}_{4}\right)_{2} \cdot 6 \mathrm{H}_{2} \mathrm{O}\right], 240 \mu \mathrm{M}$ EDTA, $2 \mathrm{~mm}$ sodium ascorbate, and $0.03 \% \mathrm{H}_{2} \mathrm{O}_{2}$ (Dixon et al. 1991). Reactions were stopped by adding $2 \mu \mathrm{l}$ of $0.2 \mathrm{M}$ EDTA and $20 \mu \mathrm{l}$ of $50 \%$ glycerol before loading on a $6 \%$ nondenaturing polyacrylamide gel. Bound complexes were detected by autoradiography, excised from the gel, eluted and then precipitated. Samples were electrophoresed through $20 \%$ sequencing gels.

\section{Quantitation}

$\beta$-Particle emission from ${ }^{32} \mathrm{P}$-labeled DNA in polyacrylamide gels was measured using a Molecular Dynamics PhosphorImager with ImageQuaNT software. Autoradiogram images were digitized using an Epson GT-6500 scanner and analysed using Image National Institutes of Health software (Dixon et al. 1991). DNA in agarose gels was stained with Sybr Green and quantified on a Molecular Dynamics FluorImager using ImageQuaNT software.

\section{Acknowledgments}

We would like to thank our colleagues, past and present, in the Microbiology Unit, Department of Biochemistry, University of Oxford, and Laboratory of Genetics, University of Glasgow, for their advice and discussions. This work was supported by the Medical Research Council and the Wellcome Trust.

The publication costs of this article were defrayed in part by payment of page charges. This article must therefore be hereby marked "advertisement" in accordance with 18 USC section 1734 solely to indicate this fact.

\section{References}

Abremski, K.E. and. R.H. Hoess. 1992. Evidence for a second conserved arginine residue in the integrase family of recombination proteins. Protein Eng. 5: 87-91.

Arciszewka, L.K. and D.J. Sherratt. 1995. Xer site-specific recombination in vitro. EMBO I. 14: 2112-2120.

Argos, P., A. Landy, K. Abremski, J.B. Egan, E. HaggardLjundquist, R.H. Hoess, M.L. Kahn, B. Kalionis, S.V.L. Narayana, L.S. Pierson, N. Sternberg, and J.M. Leong. 1986. The integrase family of site-specific recombinases: Regional similarities and global diversity. EMBO T. 5: 433-440.

Arthur, A. and D. Sherratt. 1979. Dissection of the transposition process: a transposon-encoded site-specific recombination system. Mol. \& Gen. Genet. 175: 267-274.

Austin, S., M. Ziese, and N. Sternberg. 1981. A novel role for site-specific recombination in maintenance of bacterial replicons. Cell 25: 729-736.

Bachman, B.J. 1972. Pedigrees of some mutant strains of Escherichia coli K12. Bacteriol. Rev. 36: 525-557.

Blakely, G.W. and D.J. Sherratt. 1994. Interactions of the sitespecific recombinases XerC and XerD with the recombination site dif. Nucleic Acids Res. 22: 5613-5620.

Blakely, G., S. Colloms, G. May, M. Burke, and D. Sherratt. 1991. Escherichia coli XerC recombinase is required for chromosomal segregation at cell division. New Biol. 3: 789798.

Blakely, G., G. May, R. McCulloch, L.K. Arciszewska, M. Burke, S.T. Lovett, and D.J. Sherratt. 1993. Two related recombinases are required for site-specific recombination at dif and cer in E. coli K12. Cell 75: 351-361. 
Boocock, M.R., J.L. Brown, and D.J. Sherratt. 1987. Topological specificity in $\mathrm{Tn} 3$ resolvase catalysis. In DNA replication and recombination (ed. T.J. Kelley and R. McMacken), pp. 703-718. Alan R. Liss, New York, NY.

Carrasco, C., K. Ramaswamy, T. Ramasubramian, and J. Golden. 1994. Anabena xisF gene encodes a developmentally regulated site-specific recombinase. Genes \& Dev. 8: 74-83.

Chen, J.-W., J. Lee, and M. Jayaram. 1992. DNA cleavage in trans by the active site tyrosine during FLP recombination: Switching protein partners before exchanging strands. Cell 69: 647-658.

Chi, T., P. Lieberman, K. Ellwood, and M. Carey. 1995. A general mechanism for transcriptional synergy by eukaryotic activators. Nature 377: 254-257.

Colloms, S.D., P. Sykora, G. Szatmari, and D.J. Sherratt. 1990. Recombination at ColEl cer requires the Escherichia coli $x e r C$ gene product, a member of the lambda integrase family of site-specific recombinases. J. Bacteriol. 172: 6973-6980.

Colloms, S.D., R. McCulloch, K. Grant, L. Neilson, and D.J. Sherratt. 1996. Xer-mediated site-specific recombination in vitro. EMBO I. 15: 1172-1181.

Conner, B.N., C. Yoon, J.L. Dickerson, and R.E. Dickerson. 1984. Helix geometry and hydration in an A-DNA tetramer: IC-C-G-G. J. Mol. Biol. 174: 663-695.

Corces, V.G. 1995. Chromatin insulators: Keeping enhancers under control. Nature 376: 462-463.

Cornet, F., I. Mortier, J. Patte, and J.-M. Louarn. 1994. Plasmid pSC101 harbors a recombination site, psi, which is able to resolve plasmid multimers and to substitute for the analogous chromosomal Escherichia coli site dif. I. Bacteriol. 176: 3188-3195.

Dixon, W.J., J.J. Hayes, J.R. Levin, M.F.Weidner, B.A. Dombroski, and T.D. Tullius. 1991. Hydroxyl radical footprinting. Methods Enzymol. 208: 380-413.

Erickson M.J. and R.J. Meyer. 1993. The origin of greater-thanunit-length plasmids generated during bacterial conjugation. Mol. Microbiol. 7: 289-298.

Futcher, A.B. 1986. Copy number amplification of the 2 micron circle plasmid of Saccharomyces cerevisiae. I. Theor. Biol. 119: 197-204.

Hiestand-Nauer, R. and S. Iida. 1983. Sequence of the site-specific recombinase gene $c i n$ and of its substrates serving in the inversion of the $\mathrm{C}$ segment of bacteriophage Pl. EMBO $J$. 2: 1733-1740.

Kim, J., C. Zweib, C. Wu, and S. Adhya. 1989. Bending of DNA by gene regulatory proteins: Construction and use of a DNA bending vector. Gene 85: 15-23.

Kimball, A.S., J. Lee, M. Jayaram, and T.D. Tullius. 1993. Sequence-specific cleavage of DNA via nucleophilic attack of hydrogen peroxide, assisted by FLP recombinase. Biochemistry 32: 4698-4701.

Klemm, P. 1986. Two regulatory fim genes, fim $B$ and fimE, control the phase variation of type I fimbriae in Escherichia coli. EMBO I. 5: 1389-1393.

Klippel, A., K. Cloppenborg, and R. Kahmann. 1988. Isolation and characterization of unusual gin mutants. EMBO J. 7: 3983-3989.

Kuempel, P.L., J.M. Henson, L. Dircks, M. Tecklenburg, and D.F. Lim. 1991. dif, a recA-independent recombination site in the terminus region of the chromosome of Escherichia coli. New. Biol. 3: 799-811.

Kuwabara, M., C. Yoon, T. Goyne, T. Thederahn, and D.S. Sigman. 1986. Nuclease activity of 1,10-phenanthroline-copper ion: Reactivity with CGCGAATTCGCG and its complexes with Netropsin and EcoRI. Biochemistry 25: 7401-7408.

Landy, A. 1993. Mechanistic and structural complexity in the site-specific recombination pathways of Int and FLP. Curr Opin. Genet. Dev. 3: 699-707.

Lane, D., P. Prentki, and M. Chandler. 1992. Use of gel retardation to analyze protein-nucleic acid interactions. Microbiol. Rev. 56: 509-528.

Leslie, N.R. and D.J. Sherratt. 1995. Site-specific recombination in the replication terminus region of Escherichia coli: Functional replacement of dif. EMBO J. 14: 1561-1570.

Mao, C., N.G. Carlson, and J.W. Little. 1994. Cooperative DNA-protein interaction: Effects of changing the spacing between adjacent binding sites. I. Mol. Biol. 235: 532-544.

McClarin, J.A., C.A. Frederick, B.-C. Wang, P. Greene, H.W. Boyer, J. Grable, and J.M. Rosenberg. 1986. Structure of the DNA-EcoRI endonuclease recognition complex at $3 \AA$ resolution. Science 234: 1526-1541.

McCulloch, R., M.E. Burke, and D.J. Sherratt. 1994. Peptidase activity of Escherichia coli aminopeptidase A is not required for its role in Xer site-specific recombination. Mol. Microbiol. 12: 241-251.

Nunes-Duby, S. E., L. Matsumoto, and A. Landy. 1987. Sitespecific recombination intermediates trapped with suicide substrates. Cell 50: 779-788.

Pan, G. and P.D. Sadowski. 1992. Ligation activity of FLP recombinase. The strand ligation activity of a site-specific recombinase using an activated DNA substrate. J. Biol. Chem. 267: 12397-12399.

Pargellis, C.A., S.E. Nunes-Duby, L. Moitoso de Vargas, and A. Landy. 1988. Suicide recombination substrates yield covalent $\lambda$ integrase-DNA complexes and lead to identification of the active site tyrosine. J. Biol. Chem. 263: 7678-7685.

Roberts, J.A. 1994. "The Xer site-specific recombination system." Ph.D. thesis. University of Glasgow, Glasgow, UK.

Sambrook, J., E.F. Fritsch, and T. Maniatis. 1989. Molecular cloning. A laboratory manual. Cold Spring Harbor Laboratory Press, Cold Spring Harbor, NY.

Sato, T., T. Samori, and Y. Kobayashi. 1990. The cisA ciston of Bacillus subtilis sporulation gene spoIVC encodes a protein homologous to a site-specific recombinase. $/$. Bacteriol. 172: 1092-1098.

Sherratt, D.J., L.K. Arciszewska, G. Blakely, S. Colloms, K. Grant, N. Leslie, and R. McCulloch. 1995. Site-specific recombination and circular chromosome segregation. Phil. Trans. R. Soc. Lond. (B) 347: 37-42.

Shulemovich, K., D.D. Dimaculangan, D. Katz, and M.A. Lazar. 1995. DNA bending by thyroid hormone receptor: Influence of half-site spacing and RXR. Nucleic Acids Res. 23: 811818.

Sigman, D.S., M.D. Kuwabara, C.-H.B. Chen, and T.W. Bruice. 1991. Nuclease activity of 1,10-phenanthroline-copper in study of protein-DNA interactions. Methods Enzymol. 208: 414-433.

Spassky, A., S. Rimsky, H. Buc, and S. Busby. 1988. Correlation between the conformation of Escherichia coli - 10 hexamer sequences and promoter strength: Use of orthophenanthroline cuprous complex as a structural index. EMBO $I$. 7: 1871-1879.

Stark, W.M. and M.R. Boocock. 1995. Topological selectivity in site-specific recombination. In Mobile genetic elements (ed. D.J. Sherratt), pp. 101-129. IRL Press, Oxford, UK.

Stark, W.M., D.J. Sherratt, and M.R. Boocock. 1989. Site-specific recombination by $\operatorname{Tn} 3$ resolvase: Topological changes in the forward and reverse reactions. Cell 58: 779-790.

Stark, W.M., M.R. Boocock, and D.J. Sherratt. 1992. Catalysis by site-specific recombinases. Trends Genet. 8: 432-439.

Stirling, C. J., G. Szatmari, G. Stewart, M.C.M. Smith, and D.J. Sherratt. 1988. The arginine repressor is essential for plas- 
mid-stabilizing site-specific recombination at the ColEl cer locus. EMBO I. 7: 4389-4395.

Stirling, C.J., S. Colloms, J.F. Collins, G. Szatmari, and D.J. Sherratt. 1989. xerB, an Escherichia coli gene required for plasmid ColE1 site-specific recombination is identical to pepA, encoding aminopeptidase A, a protein with substantial similarity to bovine lens leucine aminopeptidase. $E M B O$ J. 8: 1623-1627.

Summers, D.K. 1989. Derivatives of ColEl cer show altered topological specificity in site-specific recombination. $E M B O$ J. 8: 309-315.

Summers, D.K. and D.J. Sherratt. 1984. Multimerization of high copy number plasmids causes instability: ColE 1 encodes a determinant essential for plasmid monomerization and stability. Cell 36: 1097-1103.

Thompson, J.F. and A. Landy. 1988. Empirical estimation of protein-induced DNA bending angles: Applications to lambda site-specific recombination complexes. Nucleic Acids Res. 16: 9687-9705.

Tominaga, A., S. Ikemizu, and M. Enomoto. 1991. Site-specific recombinase genes in three shigella subgroups and nucleotide sequences of a pin $B$ gene and an invertible B segment from Shigella boydii. J. Bacteriol. 173: 4079-4087.

Tullius, T.D. and B.A. Dombroski. 1985. Iron(II) EDTA used to measure the helical twist along any DNA molecule. Science 230: 679-681.

1986. Hydroxyl radical "footprinting": High-resolution information about DNA-protein contacts and application to $\lambda$ repressor and Cro protein. Proc. Natl. Acad. Sci. 83: 54695473.

Warren, G.J. and A.J. Clark. 1980. Sequence-specific recombination of plasmid ColE1. Proc. Natl. Acad. Sci. 77: 67246728 .

Wiigerde, M., F. Grosveld, and P. Fraser. 1995. Transcription complex stability and chromatin dynamics in vivo. Nature 377: 209-213.

Wu, H.M. and D.M. Crothers. 1984. The locus of sequencedirected and protein-induced DNA bending. Nature 308: 509-513.

Zieg J. and M. Simon. 1980. Analysis of the nucleotide sequence of an invertible controlling element. Proc. Natl. Acad. Sci. 77: 4196-4200. 


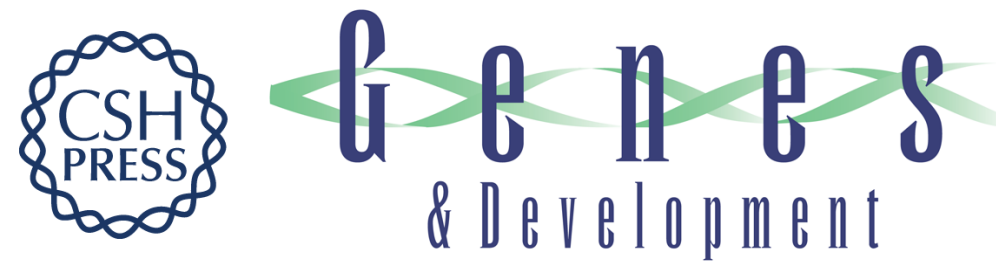

\section{Determinants of selectivity in Xer site-specific recombination.}

G Blakely and D Sherratt

Genes Dev. 1996, 10:

Access the most recent version at doi:10.1101/gad.10.6.762

$\begin{array}{ll}\text { References } & \begin{array}{l}\text { This article cites } 55 \text { articles, } 14 \text { of which can be accessed free at: } \\ \text { http://genesdev.cshlp.org/content/10/6/762.full.html\#ref-list-1 }\end{array}\end{array}$

License

Email Alerting Receive free email alerts when new articles cite this article - sign up in the box at the top Service right corner of the article or click here.

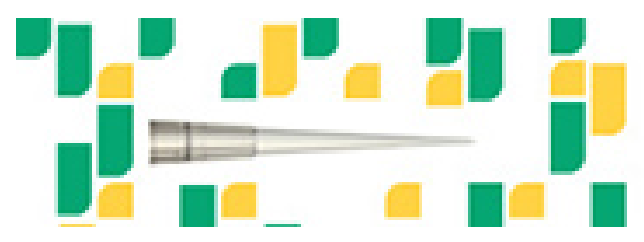

Focused on your science.

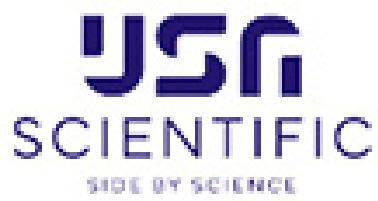

Copyright @ Cold Spring Harbor Laboratory Press 